\title{
A law of large numbers for weighted plurality
}

\author{
Joe Neeman*
}

November 14, 2018

\begin{abstract}
Consider an election between $k$ candidates in which each voter votes randomly (but not necessarily independently) and suppose that there is a single candidate that every voter prefers (in the sense that each voter is more likely to vote for this special candidate than any other candidate). Suppose we have a voting rule that takes all of the votes and produces a single outcome and suppose that each individual voter has little effect on the outcome of the voting rule. If the voting rule is a weighted plurality, then we show that with high probability, the preferred candidate will win the election. Conversely, we show that this statement fails for all other reasonable voting rules.

This result is an extension of one by Häggström, Kalai and Mossel, who proved the above in the case $k=2$.
\end{abstract}

\section{Introduction}

For elections between two candidates, it is well known that voting rules in which every voter has a small effect are good rules in the sense that they "aggregate information well:" if every voter has a small bias towards the same candidate then that candidate will win with overwhelming probability. When voters vote independently, this fact was noted by Margulis [4] and Russo [5], whose results were later strengthened by Kahn, Kalai and Linial [3] and by Talagrand [6].

When the voters are not independent, the situation is more complicated. It is no longer true, then, that every reasonable voting rule aggregates well. In fact, 2. show that if we want the aggregation to hold for every distribution of the voters, then weighted majority functions are the only option. We extend their result to the non-binary case.

The author would like to thank Elchanan Mossel for suggesting this problem and providing fruitful discussions.

\footnotetext{
${ }^{*}$ Department of Statistics, U.C. Berkeley. joeneeman@gmail.com
} 


\section{Definitions and results}

In the introduction, we made a few allusions to "reasonable" voting rules. Let us now say precisely what that means: we will require that our voting rules do not have a built-in preference for any alternative. This is a common assumption, and its definition is standard (see, eg. [1]). In what follows, the notation $[k]$ stands for the set $\{0, \ldots, k-1\}$.

Definition 2.1. A function $f:[k]^{n} \rightarrow[k]$ is neutral if $f(\sigma(x))=\sigma(f(x))$ for all $x \in[k]^{n}$ and all permutations $\sigma$ on $[k]$, where $\sigma(x)_{i}=\sigma\left(x_{i}\right)$.

Note that in the case $k=2$, a function is neutral if, and only if, it is antisymmetric according to the definition in [2].

\section{Example 2.2}

When $k=2$ and $n$ is odd, then the simple majority function (for which $f(x)=1$ if $\#\left\{i: x_{i}=1\right\}>\#\left\{i: x_{i}=0\right\}$ ) is neutral. On the other hand, if $n$ is even then in order to fully specify the simple majority function, we need to say what happens in the case of a tie; the choice of tie-breaking rule will determine whether the resulting function is neutral. For example, if we define $f(x)=x_{1}$ for every tied configuration $x$, then $f$ is neutral. On the other hand, if $f(x)=1$ for every tied configuration $x$, then $f$ is not neutral.

The example can be extended to $k \geq 3$. In this case, consider the tie-breaking rule $f(x)=x_{i}$ where $i$ is the smallest possible number for which $x_{i}$ is equal to one of the tied alternatives. This tie-breaking rule is neutral, and it is more natural than setting $f(x)=x_{1}$ because it guarantees that the output of $f$ is one of the tied alternatives.

\subsection{Weighted plurality functions}

Let us say precisely what we mean by a weighted plurality function. The definition that we take here generalizes the definition from [2] of a weighted majority function.

Definition 2.3. A function $f:[k]^{n} \rightarrow[k]$ is a weighted plurality function if there exist weights $w_{1}, \ldots, w_{n} \in \mathbb{R}_{\geq 0}$ such that $\sum_{i} w_{i}=1$ and for all $a, b \in[k]$, $f(x)=a$ implies that

$$
\sum_{i: x_{i}=a} w_{i} \geq \sum_{i: x_{i}=b} w_{i}
$$

Note that the above definition does not prescribe a particular behavior if a tie occurs between two alternatives. If the weights are chosen so that ties never occur, then the weighted plurality function is clearly neutral. Moreover, for any set of weights we can construct a neutral weighted plurality function with those weights by following the tie-breaking rule outlined in Example 2.2. 


\subsection{The influence of a voter}

The final notion that we need before stating our result is a way to quantify the power of a single voter. When $k=2$, the notion of effect is well-established and can be found, for example, in [2]. However, there does not seem to be a well-established way of quantifying the effect of voters for non-binary social choice functions. Here, we propose a definition that closely resembles the one used in 2 for binary functions.

Definition 2.4. Let $f$ be a function $[k]^{n} \rightarrow[k]$ and fix a probability distribution $P$ on $[k]^{n}$. The effect of voter $i$ is

$$
e_{i}(f, P)=\sum_{j=1}^{k} P\left(f(X)=j \mid X_{i}=j\right)-P\left(f(X)=j \mid X_{i} \neq j\right),
$$

where $X$ is a random variable distributed according to $P$.

Note that for the case $k=2$, the preceding definition reduces to

$$
e_{i}(f, P)=2\left(P\left(f(X)=1 \mid X_{i}=1\right)-P\left(f(X)=1 \mid X_{i}=0\right)\right),
$$

which is just twice the definition in [2] of a voter's effect. Also, the effect is closely related to the correlation between the voters and the outcome:

$$
\begin{aligned}
P\left(f(X)=j \mid X_{i}=j\right)-P\left(f(X)=j \mid X_{i} \neq j\right) & =\frac{\operatorname{Cov}\left(\mathbb{1}_{\{f=j\}}, \mathbb{1}_{\left\{X_{i}=j\right\}}\right)}{P\left(X_{i}=j\right) P\left(X_{i} \neq j\right)} \\
& \geq 4 \operatorname{Cov}\left(\mathbb{1}_{\{f=j\}}, \mathbb{1}_{\left\{X_{i}=j\right\}}\right)
\end{aligned}
$$

and so

$$
e_{i}(f, P) \geq 4 \sum_{j} \operatorname{Cov}\left(\mathbb{1}_{\{f=j\}}, \mathbb{1}_{\left\{X_{i}=j\right\}}\right) .
$$

\section{Example 2.5}

The simplest example of $e_{i}(f, P)$ is when $P$ is a product measure (ie. the $X_{i}$ are independent) and the function $f$ does not depend on its $i$ th coordinate; in that case, $P\left(f(X)=j \mid X_{i}=j\right)=P\left(f(X)=j \mid X_{i} \neq j\right)$ for all $j$ and so $e_{i}(f, P)=0$. On the other hand, if $P$ is a distribution such that $X_{1}=X_{2}=\cdots=X_{n}$ with probability 1 , and if $f$ is a plurality function, then $P\left(f(X)=j \mid X_{i}=j\right)=1$ for all $j$, while $P\left(f(X)=j \mid X_{i} \neq j\right)=0$; hence, $e_{i}(f, P)=1$ for all $i$.

For a less trivial example, suppose that the $X_{i}$ are independent and uniformly distributed on $[k]$. Let $f$ be an unweighted plurality function. Then the Central Limit Theorem implies that $e_{i}(f, P)=O\left(\frac{1}{\sqrt{n}}\right)$ as $n \rightarrow \infty$.

On the other hand, suppose that $f$ is still an unweighted plurality function and the $X_{i}$ are independent, but now $P\left(X_{i}=1\right)>P\left(X_{i}=j\right)+\delta$ for some $\delta>0$ and all $j \neq 1$. Then Hoeffding's inequality implies that $P\left(f(X)=1 \mid X_{i}\right) \geq$ $1-2 \exp \left(-\delta^{2} n / 4\right)$ for sufficiently large $n$, regardless of the value of $X_{i}$. In particular, this implies that $e_{i}(f, P)=O\left(\exp \left(-\delta^{2} n / 4\right)\right)$. Compared to the case where the $X_{i}$ are uniformly distributed, this demonstrates that $e_{i}(f, P)$ can depend strongly on $P$, even when $P$ is restricted to being a product measure. 


\subsection{The main result}

Our main theorem is the following:

Theorem 2.6. (a) For every $\delta>0$ and $\epsilon>0$, there is a $\tau>0$ such that for every weighted plurality function $f$ with weights $w_{i}$ and every probability distribution $P$ on $[k]^{n}$, if $e_{i}(f, P) \leq \tau$ and there is a set $A \subset[n]$ such that $\sum_{i} w_{i} P\left(X_{i}=a\right) \geq \sum_{i} w_{i} P\left(X_{i}=b\right)+\delta$ for all $i \in[n]$, all $a \in A$ and all $b \notin A$, then $P(f(X) \in A) \geq 1-\epsilon$.

(b) If $f$ is not a weighted plurality function then there exists a probability distribution $P$ on $[k]^{n}$ such that $P\left(X_{i}=2\right)>P\left(X_{i}=1\right)$ for all $i \in[n]$ but $P(f(X)=1)=1$ (and hence $e_{i}(f, P)=0$ for all $\left.i\right)$.

We remark that the Theorem is constructive in the sense that we can give an algorithm (based on solving a linear program) which either constructs some weights $w_{i}$ witnessing the fact that $f$ is a weighted plurality, or a probability distribution $P$ satisfying part (b).

Parts (a) and (b) of Theorem 2.6 are converse to one another in the following sense: under the hypothesis of small effects, part (a) says that if there is a gap between the popularity of the most popular alternatives $A$ and the less popular alternatives $A^{c}$ then a weighted plurality function will choose an alternative in $A$. Part (b) shows that this property fails for every function that is not a weighted plurality. Note that part (a) has an important special case, which is closer to the statement of [2]: if $P\left(X_{i}=a\right) \geq P\left(X_{i}=b\right)+\delta$ for all $i \in[n]$ and all $b \neq a$, then $f(X)=a$ with high probability if the effects are small enough.

The remainder of the paper is devoted to the proof of Theorem 2.6.

Proof of Theorem [2.6 (a). This part of the proof follows very closely the argument in [2]. Suppose that $f$ is a weighted plurality function with weights $w_{i}$. The first step is to show that $f$ is "correlated" in some sense with each voter: define $p_{i j}=P\left(X_{i}=j\right)$ and let $W_{j}$ be the (random) weight assigned to alternative $j: W_{j}=\sum_{i: X_{i}=j} w_{i}$. Then

$$
\begin{aligned}
& \mathbb{E} \sum_{i=1}^{n} w_{i} \sum_{j=1}^{k} \mathbb{1}_{\{f(X)=j\}}\left(\mathbb{1}_{\left\{X_{i}=j\right\}}-p_{i j}\right) \\
& =\mathbb{E}\left(\sum_{i, j} w_{i} \mathbb{1}_{\{f(X)=j\}} \mathbb{1}_{\left\{X_{i}=j\right\}}-\sum_{i, j} \mathbb{1}_{\{f(X)=j\}} w_{i} p_{i j}\right) \\
& =\mathbb{E} \sum_{i, j} w_{i} \mathbb{1}_{\{f(X)=j\}} \mathbb{1}_{\left\{X_{i}=j\right\}}-\sum_{j} P(f=j) \mathbb{E} W_{j} .
\end{aligned}
$$

Now, let $\alpha_{j}=P(f=j)$ and set $\tilde{\alpha}_{j}=\alpha_{j} /\left(\sum_{i \in A} \alpha_{i}\right)$ for $j \in A$ and $\tilde{\alpha}_{j}=0$ otherwise. The first term of (11) is just

$$
\begin{aligned}
\mathbb{E} \sum_{i, j} w_{i} \mathbb{1}_{\{f(X)=j\}} \mathbb{1}_{\left\{X_{i}=j\right\}}=\mathbb{E} \sum_{j} \mathbb{1}_{\{f(X)=j\}} W_{j} \\
\geq \mathbb{E} \sum_{j} \mathbb{1}_{\{f(X)=j\}} \sum_{i} \tilde{\alpha}_{i} W_{i}=\sum_{i} \tilde{\alpha}_{i} \mathbb{E} W_{j}
\end{aligned}
$$


since the winning alternative always has at least as much weight as any convex combination of alternatives. Since $\min _{j \in A} \mathbb{E} W_{j} \geq \max _{j \notin A} \mathbb{E} W_{j}+\delta$, we can plug (2) into (11) to obtain

$$
\text { (11) } \begin{aligned}
& \geq \sum_{j} \tilde{\alpha}_{j} \mathbb{E} W_{j}-\sum_{j} \alpha_{j} \mathbb{E} W_{j} \\
& \geq \sum_{j \in A}\left(\tilde{\alpha}_{j}-\alpha_{j}\right) \delta \\
& =\delta P(f \notin A) .
\end{aligned}
$$

Recalling that $e_{i}(f, P) \geq 4 \sum_{j} \operatorname{Cov}\left(\mathbb{1}_{\{f=j\}}, \mathbb{1}_{\left\{X_{i}=j\right\}}\right)$, we have

$$
\begin{aligned}
\delta P(f \notin A) & \leq \mathbb{E} \sum_{i=1}^{n} w_{i} \sum_{j=1}^{k} \mathbb{1}_{\{f(X)=j\}}\left(\mathbb{1}_{\left\{X_{i}=j\right\}}-p_{i j}\right) \\
& \leq \frac{1}{4} \sum_{i} w_{i} e_{i}(f, P) \\
& \leq \frac{\tau}{4}
\end{aligned}
$$

and so one direction of the theorem is proved once we take $\tau$ small enough that $\epsilon \geq \tau /(4 \delta)$.

The proof of the second part of the theorem follows the idea of [2], in that we use linear programming duality to find a witness for $f$ being a weighted plurality function. However, the details of the proof are quite different, since [2] uses a well-known linear program (the fractional vertex cover of a hypergraph) which does not extend beyond $k=2$.

The proof idea is this: we will write down a linear program and its dual. If the primal program has a large enough value it will turn out that $f$ is a weighted plurality function. Otherwise, the dual has a small value and the dual variables witness the claim of Theorem 2.6(b). In particular, note that this proof provides the algorithm that we mentioned after the statement of Theorem 2.6.

First we make a trivial observation that will simplify our linear program considerably: if a function is neutral, it is easier to check whether it is a weighted plurality because it is not necessary to try all possible combinations of $a, b \in[k]$ :

Proposition 2.7. Suppose $f:[k]^{n} \rightarrow[k]$ is neutral. Then $f$ is a weighted plurality if and only if there exist weights $w_{1}, \ldots, w_{n} \in \mathbb{R}$ such that $f(x)=1$ implies that

$$
\sum_{i: x_{i}=1} w_{i} \geq \sum_{i: x_{i}=2} w_{i}
$$

We can write a linear program for checking whether a given neutral function $f$ is a weighted plurality. The variables for this program are $t ; w_{i}$ for each $i \in[n]$ and $g_{x}$ for each $x \in[k]^{n}$ for which $f(x)=1$. In standard form, the primal 
program is the following:

$$
\begin{aligned}
\operatorname{maximize} & t_{+}-t_{-} \\
\text {subject to } & g_{x} \geq 0 \text { for all } x \in[k]^{n} \text { such that } f(x)=1 \\
& w_{i} \geq 0 \text { for all } i \in[n] \\
& t_{+} \geq 0 \text { and } t_{-} \geq 0 \\
& \sum_{i} w_{i}=1 \\
& \sum_{i: x_{i}=1} w_{i}-\sum_{i: x_{i}=2} w_{i}-g_{x}-\left(t_{+}-t_{-}\right)=0 \text { for all } x \in[k]^{n} \text { with } f(x)=1 .
\end{aligned}
$$

Proposition 2.8. Let $t^{*}$ be the value of the above linear program. If $t^{*} \geq 0$ then $f$ is a weighted plurality function.

Proof. Let $w_{i}, g_{x}, t_{+}$and $t_{-}$be feasible points such that $t_{+}-t_{-} \geq 0$. Then, for all $x$ with $f(x)=1$,

$$
\sum_{i: x_{i}=1} w_{i}-\sum_{i: x_{i}=2} w_{i}=g_{x}+\left(t_{+}-t_{-}\right) \geq 0
$$

and so $f$ satisfies the conditions of Proposition 2.7

Now consider the dual program; since the primal is in standard form, the dual is easy to write down. Let the dual variables be $a$ and $q_{x}$ for all $x$ such that $f(x)=1$. Then the dual program is:

$$
\begin{array}{ll}
\text { minimize } & a_{+}-a_{-} \\
\text {subject to } & \sum_{x: f(x)=1} q_{x} \leq-1 \\
& \sum_{x: f(x)=1}\left(\mathbb{1}_{\left\{x_{i}=1\right\}}-\mathbb{1}_{\left\{x_{i}=2\right\}}\right) q_{x}+\left(a_{+}-a_{-}\right) \geq 0 \text { for all } i \in[n] \\
& q_{x} \leq 0 \text { for all } x \text { such that } f(x)=1 \\
& a_{+} \leq 0 \text { and } a_{-} \leq 0 .
\end{array}
$$

Proposition 2.9. Let $a^{*}$ be the value of the above dual program. If $a^{*}<0$ then there exists a probability distribution on $[k]^{n}$ such that $P\left(X_{i}=2\right)>P\left(X_{i}=1\right)$ for all $i$ but $f(X)=1$ almost surely.

Proof. Choose a feasible point with $a_{+}-a_{-}<0$ and define $p_{x}=-q_{x} /\left(\sum_{x} q_{x}\right)$. Then $p_{x} \geq 0$ and $\sum_{x} p_{x}=1$, so we can define a probability distribution by $P(X=x)=p_{x}$ when $f(x)=1$ and $P(X=x)=0$ otherwise. Under this distribution, $f(X)=1$ with probability 1 . On the other hand, with $a_{+}-a_{-}<0$ the constraints of the dual program imply that

$$
\sum_{x: f(x)=1} \mathbb{1}_{\left\{x_{i}=1\right\}} q_{x}>\sum_{x: f(x)=1} \mathbb{1}_{\left\{x_{i}=2\right\}} q_{x}
$$


for all $i$. Thus,

$$
P\left(X_{i}=1\right)=\sum_{x: f(x)=1} \mathbb{1}_{\left\{x_{i}=1\right\}} p_{x}<\sum_{x: f(x)=1} \mathbb{1}_{\left\{x_{i}=2\right\}} p_{x}=P\left(X_{i}=2\right)
$$

for all $i$.

To conclude the proof of Theorem 2.6. note that both the primal and dual programs are feasible and bounded and so $a^{*}=t^{*}$.

\section{References}

[1] S.J. Brams and P.C. Fishburn. Voting procedures. Handbook of social choice and welfare, 1:173-236, 2002.

[2] O. Häggström, G. Kalai, and E. Mossel. A law of large numbers for weighted majority. Advances in Applied Mathematics, 37(1):112-123, 2006.

[3] J. Kahn, G. Kalai, and N. Linial. The influence of variables on Boolean functions. In Proceedings of the 29th Annual Symposium on Foundations of Computer Science, pages 68-80. IEEE Computer Society, 1988.

[4] G. Margulis. Probabilistic characteristic of graphs with large connectivity. Problems Info. Transmission, 10:174-179, 1977.

[5] L. Russo. An approximate zero-one law. Probability Theory and Related Fields, 61(1):129-139, 1982.

[6] M. Talagrand. On Russo's approximate zero-one law. The Annals of Probability, 22(3):1576-1587, 1994. 